\title{
Neighboring Atom Collisions in Solid-State High Harmonic Generation
}

\author{
Ruixin Zuo, ${ }^{1,2}$ Alexander Trautmann, ${ }^{3}$ Guifang Wang, ${ }^{1}$ Wolf-Rüdiger Hannes $\left(\mathbb{D},{ }^{3}\right.$ \\ Shidong Yang, ${ }^{1,2}$ Xiaohong Song $\mathbb{D}^{1,2,4}$ Torsten Meier $\mathbb{D}^{3}{ }^{3}$ Marcelo Ciappina $\mathbb{D}^{5,6}$ \\ Huynh Thanh Duc, ${ }^{7}$ and Weifeng Yang $\mathbb{D}^{1,2,4}$
}

${ }^{1}$ Research Center for Advanced Optics and Photoelectronics, Department of Physics, College of Science, Shantou University, Shantou, Guangdong 515063, China

${ }^{2}$ Institute of Mathematics, Shantou University, Shantou, Guangdong 515063, China

${ }^{3}$ Department of Physics and Center for Optoelectronics and Photonics Paderborn (CeOPP), University of Paderborn, Warburger Str. 100, D-33098 Paderborn, Germany

${ }^{4}$ Key Laboratory of Intelligent Manufacturing Technology of MOE, Shantou University, Shantou, Guangdong 515063, China

${ }^{5}$ Physics Program, Guangdong Technion-Israel Institute of Technology, Shantou, Guangdong 515063, China

${ }^{6}$ Technion-Israel Institute of Technology, Haifa 32000, Israel

${ }^{7}$ Ho Chi Minh City Institute of Physics, Vietnam Academy of Science and Technology, 1 Mac Dinh Chi, District 1, Ho Chi Minh City, Vietnam

Correspondence should be addressed to Xiaohong Song; songxh@stu.edu.cn, Torsten Meier; torsten.meier@uni-paderborn.de, and Weifeng Yang; wfyang@stu.edu.cn

Received 4 May 2021; Accepted 23 August 2021; Published 1 November 2021

Copyright (c) 2021 Ruixin Zuo et al. Exclusive Licensee Xi'an Institute of Optics and Precision Mechanics. Distributed under a Creative Commons Attribution License (CC BY 4.0).

\begin{abstract}
High harmonic generation (HHG) from solids shows great application prospects in compact short-wavelength light sources and as a tool for imaging the dynamics in crystals with subnanometer spatial and attosecond temporal resolution. However, the underlying collision dynamics behind solid HHG is still intensively debated and no direct mapping relationship between the collision dynamics with band structure has been built. Here, we show that the electron and its associated hole can be elastically scattered by neighboring atoms when their wavelength approaches the atomic size. We reveal that the elastic scattering of electron/hole from neighboring atoms can dramatically influence the electron recombination with its left-behind hole, which turns out to be the fundamental reason for the anisotropic interband HHG observed recently in bulk crystals. Our findings link the electron/hole backward scattering with Van Hove singularities and forward scattering with critical lines in the band structure and thus build a clear mapping between the band structure and the harmonic spectrum. Our work provides a unifying picture for several seemingly unrelated experimental observations and theoretical predictions, including the anisotropic harmonic emission in $\mathrm{MgO}$, the atomic-like recollision mechanism of solid HHG, and the delocalization of HHG in $\mathrm{ZnO}$. This strongly improved understanding will pave the way for controlling the solid-state HHG and visualizing the structure-dependent electron dynamics in solids.
\end{abstract}

\section{Introduction}

Collision dynamics lies at the heart of particle physics and also constitutes one of the fundamental blocks in strongfield physics and attosecond science [1-5]. When an intense laser field interacts with an atom, electron can be liberated and then accelerated by the oscillating laser field in the continuum. When the electric field reverses its direction, the electron could be driven back to collide with the atomic core. During the recollision, if the electron recombines with the parent ion, high-order harmonics (HHG) and attosecond photon bursts would be emitted; otherwise, the electron would be scattered away and high-energy electrons would be detected. The emitted high-energy photons and electrons encode structural and dynamical information about the atom or molecule they have left behind. Therefore, the 
electron recollision with the ion/molecular core provides not only an efficient way to generate attosecond optical and electron pulses but also an optical technique to probe the structure and ultrafast electronic dynamics in its natural time scale [6-11].

Recently, the recollision model has been transferred to solids, where the electron-hole recombination has been considered as the main mechanism of interband solid HHG [12-20]. However, in contrast to atomic HHG, where the liberated electron moves in the continuum and the influence of the ion core is negligible when the electron travels far away, in solid HHG, the electron and the leftbehind hole move in periodic potentials, making collisions with other neighboring atoms in the lattice highly probable. In this way, the microscopic electron dynamics becomes much more intricate [21-29]. Thus, new fundamental questions have to be raised: Can the electron recombine with other uncorrelated hole at the positions of neighboring atoms or is it scattered away during a collision with neighboring atoms? Under what circumstances can such collisions occur and how would they affect the interband solid-state $\mathrm{HHG}$ ?

Here, we focus on resolving these questions. Our results support the fact that the electron does not directly recombine with but is scattered by the neighboring atoms. When driven by a linearly polarized laser field, the backward scattering of electron/hole by neighboring atoms would increase the rate of the recombination of electron with its associated hole, whereas the forward scattering would suppress it. This mechanism represents the principal reason of the anisotropy of solid-state HHG. Moreover, we clarify that delocalization of HHG in solids can be attributed to the fact that the electron and its left-behind hole experience different times of backward scattering. In this way, they recombine at atomic sites which are different from the ones where they were born. Most importantly, we identify a close correspondence between the Van Hove singularity points in the band structures and the backward scattering of electron/hole from neighboring atoms and map these effects onto the harmonic spectrum.

\section{Materials and Methods}

To unveil the collision dynamics of electron/hole with other atoms in solids, we focus on the recently observed anisotropic HHG in MgO reported in Reference [21]. The experimental harmonic spectra from $\mathrm{MgO}$ reveal that interband current plays a dominant role and the interband high harmonics show a clear fourfold symmetry with a set of sharp maxima along $0^{\circ}, 90^{\circ}, 180^{\circ}$, and $270^{\circ}$, which are the $\mathrm{Mg}-\mathrm{O}$ $\langle 100\rangle$ nearest-neighbor bonding directions. A second set of maxima can be seen along $45^{\circ}, 135^{\circ}, 225^{\circ}$, and $315^{\circ}$, which are the $\mathrm{Mg}-\mathrm{Mg}$ (or $\mathrm{O}-\mathrm{O}$ ) $\langle 110\rangle$ nearest-neighbor directions. The enhancement (diminution) of harmonic efficiency was attributed to the connection (avoiding) of the semiclassical electron trajectories with the neighboring atomic sites, which suggests that the electron would not necessarily recombine with its associated hole to emit high-frequency radiation $[21,22]$. Subsequent investigation about delocalization of $\mathrm{HHG}$ in $\mathrm{ZnO}$ predicted by a Wannier-Bloch approach, whereby an electron ionized from one site in the periodic lattice may recombine at another one, seems to support this viewpoint [23]. Very recently, similar angular dependence of HHG has also been observed in Reference [30] (see Figure 2 of the supplementary information in [30]), where the angular dependence of the enhancement of HHG was attributed to an enhanced constructive interference of the interband currents at Van Hove singularities.

Here, we perform first full quantum theoretical simulations based on semiconductor Bloch equations (SBEs) including intra- and interband dynamics $[15,31]$ (the mathematical details are described in Supplementary Materials (available here)). The band structure and the eigenfunctions are calculated by the density functional theory (DFT) package in ELK [32], and the smooth transition dipole phase is gotten in the twisted parallel transport gauge [33-35]. In $\mathrm{MgO}$, the real parts of the complex-valued transition dipole moment (TDM) are close to 0 , whereas the imaginary parts have both positive and negative values; i.e., the phases are 0 or $\pi$, and as a result, the accumulated Berry phase is equal to 0 , which is consistent with Reference [35]. The imaginary parts of TDM are shown in Figure S1 in Supplementary Materials. The driving laser wavelength we used in our simulation is $1.3 \mu \mathrm{m}$, the same as in the experiments [21, 30]. The pulse duration is 10 laser cycles and the peak field amplitude is $1.2 \mathrm{~V}^{-1}$, which are chosen to correspond to the experimental conditions. The dephasing time $T_{2}=T_{0} / 4$, where $T_{0}$ is the laser cycle. In Figure 1, the left part (i.e., from $90^{\circ}$ to $270^{\circ}$ ) is the experimental result extracted from Reference [21] and the right part (i.e., from $-90^{\circ}$ to $90^{\circ}$ ) is our quantum simulation result. The anisotropy of $\mathrm{HHG}$ observed in experiments is well reproduced by our numerical simulation.

\section{Results and Discussion}

3.1. Subcycle Dynamics of Orientation-Dependent HHG. The purpose of this work is to provide a clear physical understanding of the subcycle collision dynamics in solids. To this end, in the following, we confine all the excitation and emission dynamics within one laser cycle by adopting a near single-cycle optical pulse (see blue dashed line shown in Figures 2(b) and 2(e)). Such pulses have been successfully synthesized in the terahertz, mid-infrared, visible, and UV spectral range [36-38]. Their potential for subcycle electron control in solids and for tracking the nonlinear response of bound electrons has been demonstrated recently [37]. Following Reference [21], $\theta$ is defined as the angle between the polarization direction and the cubic ( $\mathrm{Mg}-\mathrm{O}$ bonding) direction of the crystal.

We focus on the interband dynamics which dominate the emission above the band gap in MgO. Figures 2(a)-2(c) show the time-frequency analysis of the time-dependent interband polarization for $\theta=0^{\circ}, 27^{\circ}$, and $45^{\circ}$, respectively, in the considered spectral region. The results are compatible with the experimental and quantum simulation results when driven by a long-duration laser field (see Figure 1), where the high- 


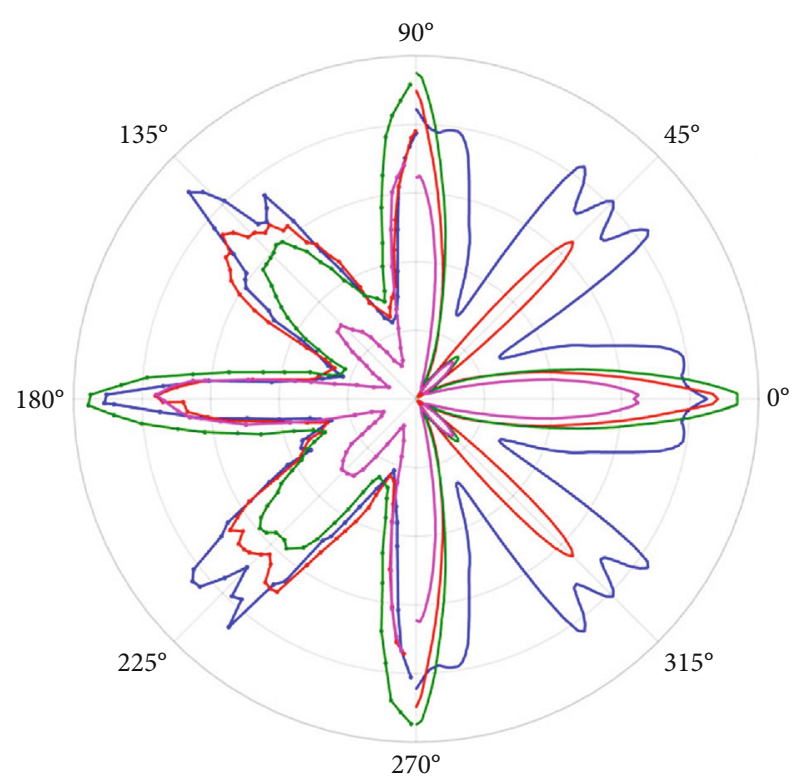

Experiment

$\rightarrow 13$ th $(\times 146)$

$\because 15$ th $(\times 8)$

$\longrightarrow 17$ th

$\longrightarrow 19$ th

Theory

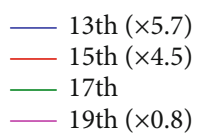

Figure 1: Comparison of experimental data (lines with points on the left), extracted from Reference [21], and simulated HHG (solid lines on the right) for $\mathrm{MgO}$.

energy parts of the harmonics are strongest along $\theta=0^{\circ}$, join with a secondary strong lobe along $\theta=45^{\circ}$, and nearly disappear along around $\theta=27^{\circ}[21,30]$. This behavior demonstrates that the angular dependence of the HHG observed in the experiments is essentially related to dynamics at a subcycle time scale. Recently, the orientation dependence for the highest harmonics (21st) has been discussed in Reference [39] and was interpreted in the momentum space picture in terms of the several different conduction band contributions. Here, we mainly focus on the lower-order harmonics, those in the primary plateau, and analyze their underlying physics in the real space picture.

3.2. HHG Assisted and Suppressed by Scattering with Neighboring Atoms. To explore the subcycle collision dynamics, we calculate the real-space electron and hole trajectories for the same laser and structural parameters as that in Figures 2(a)-2(c), with a semiclassical analysis and focus on electrons that can reencounter with their associated holes (see Supplementary Materials for further details (available here)) $[15,16]$. We consider the electron-hole pair is created at the $\Gamma$ point, where the transition dipole moment peaks. The dashed and solid lines in Figures 2(d)-2(f) show the harmonic photon energy (i.e., the energy difference of the electron-hole pair at the momentum where they reencounter with each other) as a function of the time of the electronhole pair creation (ionization, dashed lines) and recombination (emission, solid lines), respectively. Because the total interband harmonic spectrum extracted from the quantum simulation (shown in Figures 2(a)-2(c)) is a result of a coherent superposition of all electron trajectories ionized at different crystal momentum in the Brillouin zone (BZ), for better comparison with the semiclassical simulation, we anatomize the total interband $\mathrm{HHG}$ and extract the contribution of electron/hole trajectories ionized only around the $\Gamma$ point with a reciprocal-space-trajectory (RST) method [25] (see the color maps in Figures 2(d)-2(f)). The semiclassical simulations show very good agreement with the quantum results (Figures 2(d)-2(f)), demonstrating the validity of the picture that an electron would recombine with its associated hole [15, $16]$, rather than other unrelated one $[21,22,40]$.

A key feature in the semiclassical simulations is that there are two sets of electron-hole recombination trajectories for a laser polarization along $\theta=0^{\circ}$ and $\theta=45^{\circ}$, which are referred to as " 1 " and " 2 " in Figures $3(\mathrm{~d})$ and $3(\mathrm{f})$, whereas set " 1 " disappears for $\theta=27^{\circ}$ (see Figure $3(\mathrm{e})$ ). It is striking that ionization occurs before the field crest in set " 1 " considering the associated recombination would be absent in atoms [5]. By comparing the electron/hole trajectories in momentum space (see the dashed arrow lines in Figures 3(a) and $3(\mathrm{~b})$ ) to that in real space (see Figures $3(\mathrm{~d})$ and $3(\mathrm{e})$ ) in this set, we find that the electron and hole reverse their directions in real space whenever they reach the critical points where $\nabla_{k} E_{c / v}(k)=0$ in momentum space (see black dots in Figures 3(a) and 3(b)). These critical points are known as Van Hove singularities [41, 42].

Figure 3(c) shows the real space trajectories of electron (black line) and hole (red line) for a laser polarization along $\theta=0^{\circ}$ and ionization time at $-0.13 T_{0}$. In this case, the electron and hole reverse their directions at about $t=0.08 T_{0}$ (see Figure 3(c)), much earlier than the electric field inverts its direction, which occurs at $0.25 T_{0}$. This suggests that the direction inversion of the electron and hole here is not driven by the electric field as in atoms. According to the movie of electron/hole's movement track shown in the Movie S1 and Movie S2 in Supplementary Materials, we attribute such direction inversion to the backward scattering resulting from the collision of the electron/hole with other neighboring atoms.

In a recent work [30], it has been shown that the recombination rate of semiclassical trajectories would become infinite, and thus, the semiclassical method would fail if the recombination of electron and hole occurs at the critical points where $\nabla_{k} E_{c}=\nabla_{k} E_{v}=0$. In our case, however, the results seem to demonstrate that for backward scatteringinduced electron-hole recombination, the recombination would in practice rarely occur at these singularities. This is because that, at these critical points, the electron and hole just begin to reverse their directions due to the scattering. After that, they still need to move towards each other before they can reencounter; therefore, recombination would occur around but not exactly at these singular points. This is compatible with the experimental results shown in Figure 2 of Reference [30], where the enhanced harmonics are indeed near but not exactly at the singular points, which confirms the neighboring atom scattering-assisted electron-hole recombination mechanism of $\mathrm{HHG}$ in $\mathrm{MgO}$. 

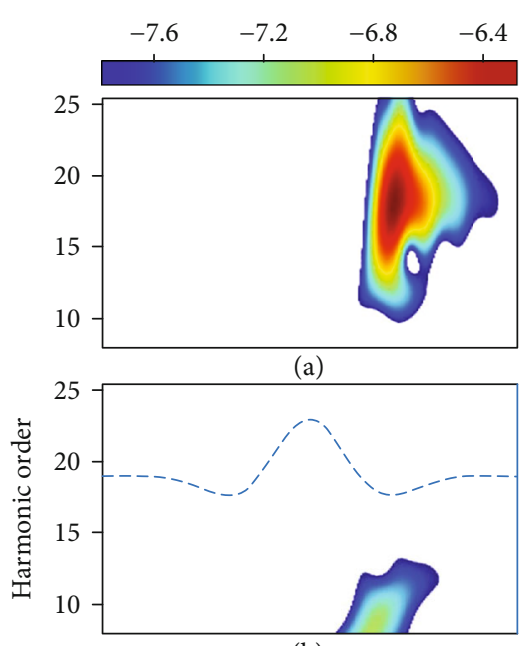

(b)

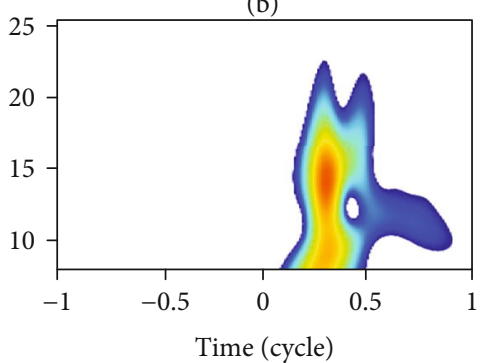

(c)

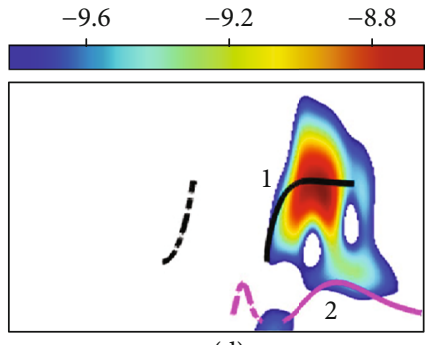

(d)

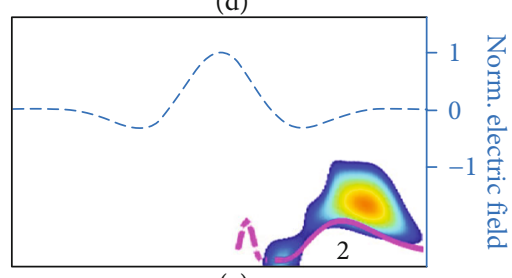

(e)

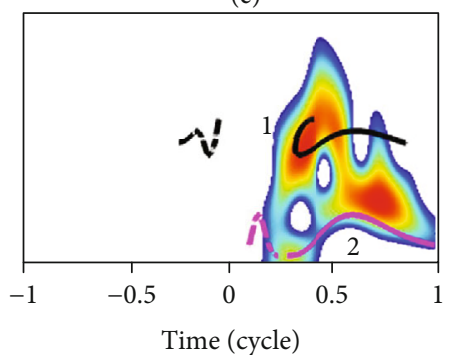

(f)

$==$ Ionization

Emission

Figure 2: Comparison between the quantum and the classical simulations for $\mathrm{MgO}$. (a-c) Time-frequency analysis of total HHG obtained from the numerical solutions of SBEs. (d-f) Time-frequency analysis of HHG only including the contribution of electron trajectories tunneling at around the $\Gamma$ point; the dashed and solid lines indicate the emitted harmonic energy as a function of ionization (dashed lines) and recombination (solid lines) times in the semiclassical simulations. All spectral intensities in color map are on a logarithmic scale. To indicate the time scale, the waveform of the incident laser electric field is shown by the blue dashed lines in (b) and (e).

Note that collision with other atoms can occur only when the wavelength of electron/hole is small enough to be comparable to atoms in size. Therefore, only critical points at high-momentum region are relevant to this scattering mechanism. Under the present laser conditions, only when ionization occurs before the field crest, the electron and hole could get enough energy such that the wave number may get large enough that scattering from other neighboring atomic sites gets relevant [43]. Otherwise, if ionization occurs after the field crest, the electron and hole would move only within a small fraction of the Brillouin zone near the $\Gamma$ point (see right panel of the Movie S3 in Supplementary Materials). Due to the small wave number, the wavelength of electron/hole is much larger than the size of atoms, so that the electron/hole would directly cross neighboring atoms as if they were absent, and no scattering with these atomic sites occurs. In this case, only after the electric field changes its sign, the electron and its associated hole would reverse their directions and recombine with each other to emit harmonic photons, which essentially is an atomic-like scenario (see set " 2 " in Figure 2). In momentum space, the direction inversion of the electron/hole occurs when they cross the $\Gamma$ point, which is also a Van Hove singularity but located at low- momentum region. The atomic-like electron-hole recombination mechanism is the main cause of the atomic-like behavior of $\mathrm{HHG}$ from $\mathrm{ZnO}$ [14-16].

Along $\theta=45^{\circ}$, there are two Van Hove singularities, in both the conduction and valence bands at highmomentum region (indicated by "P2" and "P3" in the conduction band and "P2'" and "P 3 "” in the valence band of Figures 3(a) and 3(b)), which means that multiple backward scatterings from neighboring atoms would occur before the electron recombines with its associated hole (see Figure 3(e) and Movie S2 in Supplementary Materials). Multiple scattering trajectories correspond to earlier ionization and later recombination (see Figure $2(\mathrm{f})$ ). In the classical simulation, the dephasing is not taken into account, whereas indeed a fast dephasing is included in its quantum counterpart which would attenuate the probability of recombination that takes a longer time due to multiple scattering. This is confirmed in Figure 2(f), where the emission is weaker at a later recombination time. As a result, multiple backward scattering before the electron recombination is the basic reason of a weaker HHG signal along $\theta=45^{\circ}$ orientation than that along $\theta=0^{\circ}$ in both our quantum calculation and experiments [21] (see Figure 1 and Figures 2(d) and 2(f)). 


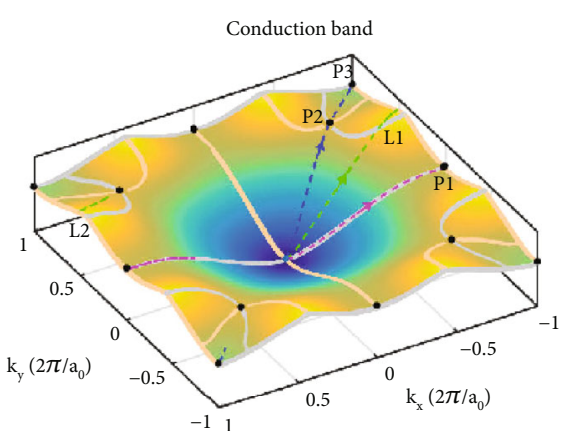

(a)

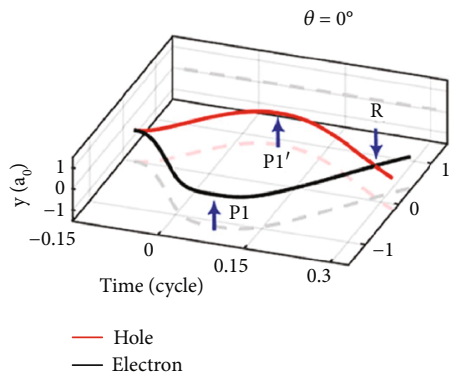

(c)

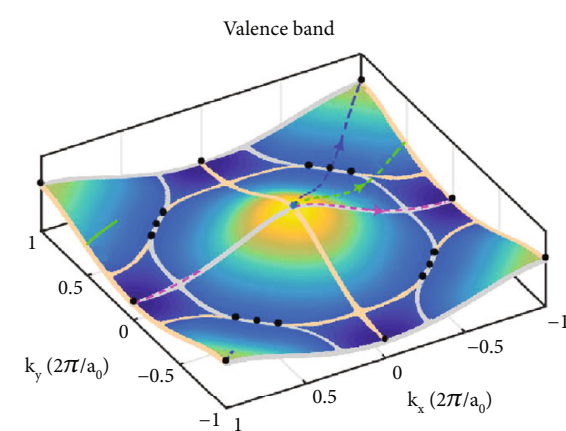

(b)

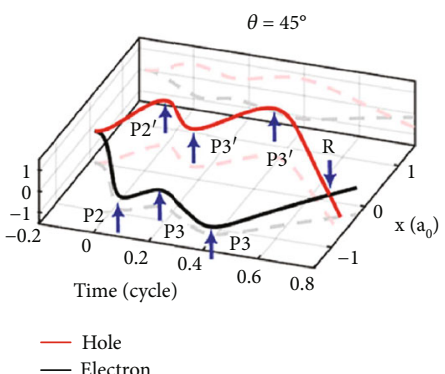

(e)

Figure 3: Mapping between the band structure and the collision dynamics. (a, b) 2D conduction and valence band structures of MgO. The black dots indicate the places where $\nabla_{k} E_{c}(k)=0$. The light yellow solid lines show where only $\nabla_{k_{x}} \varepsilon(k)=0$, and grey solid lines show only $\nabla_{k_{y}} \varepsilon(k)=0$. (c-h) The time-dependent position of electron (black) and hole (red) trajectories with an initial ionization time before the field crest for $\theta=0^{\circ}$ (c), $\theta=27^{\circ}$ (d), and $\theta=45^{\circ}$ (e). The deep pink, green, and yellow dashed arrow lines in (a) and (b) show the corresponding trajectories in momentum space for $\theta=0^{\circ}, 27^{\circ}$, and $45^{\circ}$, respectively.

The neighboring atom scattering-assisted electron-hole recombination mechanism can also explain the delocalization mechanism recently predicted for HHG emission in $\mathrm{ZnO}$ along the $\Gamma-\mathrm{M}$ direction, whereby an electron ionized from one site in the periodic lattice may recombine at others [23]. Figure 4(a) shows the band structure along the $\Gamma-\mathrm{M}$ direction of $\mathrm{ZnO}$ adopted in Reference [23]. There exists only one Van Hove singularity in the conduction band (indicated by "P1"), whereas two in the valence band (indicated by "P1'" and "P2'") in the high-momentum region. According to our scattering-assisted electron-hole recombination picture introduced above, such band structure means that the electron would experience only one backward scattering; however, the hole would experience multiple ones, if the electron-hole pair is created before the electric field peak and can reach these critical points (see Figures 4(b) and $4(\mathrm{c})$ ). Multiple scattering makes the average velocity of the hole lower than that of electron. Thus, when the electron returns to the parent atomic site where it was born, the hole is still far away. In this way, the electron will continue its motion through the parent atomic site and reencounter the hole near another atomic site (see Figure 4(b)) (more details can be seen in the Movie S4 in Supplementary Materials). Figure 4(d) shows the distance between the creation and recombination positions of an electron-hole pair in $\mathrm{ZnO}$ as a function of the ionization and recombination instants, when driven by a subcycle pulse. This result is consistent with the corresponding Wannier analysis (see Supplemen- tary Materials for further details (available here)) shown in Figure 4(e) and that presented in Reference [23], demonstrating that recombination of electron with its associated hole is the principal mechanism, and the different dynamics between the electron and the left-behind hole is the underlying physics of the delocalization of HHG in solids.

In addition to the backward scattering, forward scattering can also occur during the collision. In the momentum space, the forward scattering points correspond to some critical lines in the band structures, where only $\nabla_{k_{x}} E_{c / v}(k)=0$ (light yellow solid lines in Figures 3(a) and 3(b)) or $\nabla_{k_{v}} E_{c / v}$ $(k)=0$ (grey solid lines in Figures $3(\mathrm{a})$ and $3(\mathrm{~b})$ ). When the laser polarization is linearly polarized along $\theta=27^{\circ}$ in $\mathrm{MgO}$, the trajectories in the momentum space indicate that the electron and its associated hole born before the field crest would go through some critical lines (see green dashed arrow lines in Figures 3(a) and 3(b)). The comparison between the trajectories of electron and hole in real and momentum space reveals that, whenever the electron/hole crosses a critical line in momentum space, the electron/hole trajectory in real space would experience a forward scattering by a side collision with other neighboring atoms. This happens when the electron/hole reverses its direction along one direction component while keeps on moving along the other (see Figures 3(a), 3(b), and 3(d) and more details are provided in Movie S5 in Supplementary Materials). After experiencing several such side collisions, the electron is farther and farther away from its associated hole and recombination can hardly 


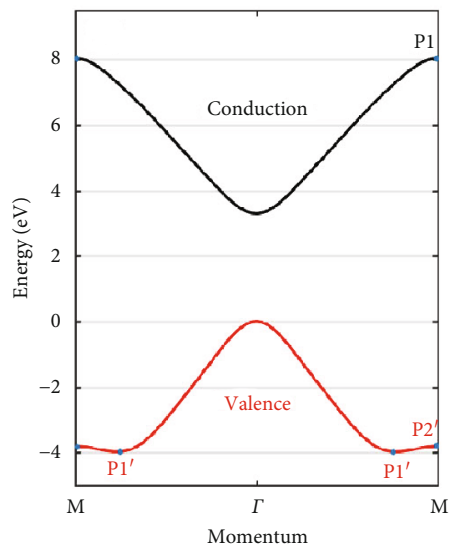

(a)

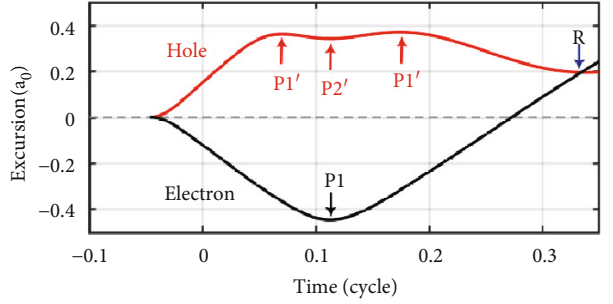

(b)

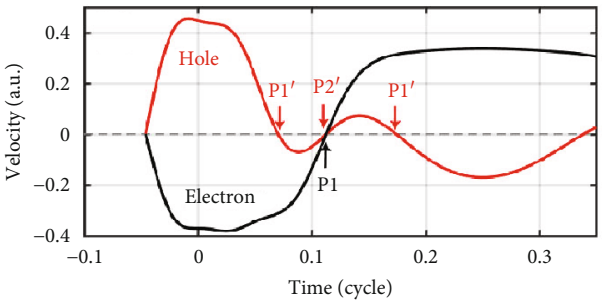

(c)

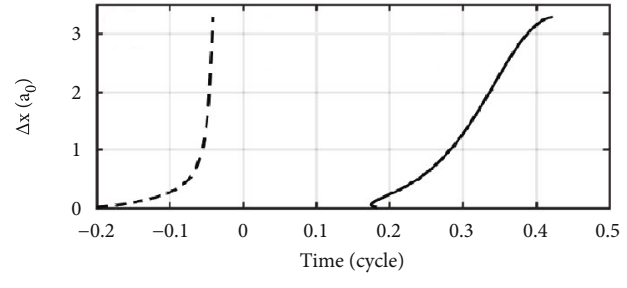
- Ionization
- Recombination

(d)

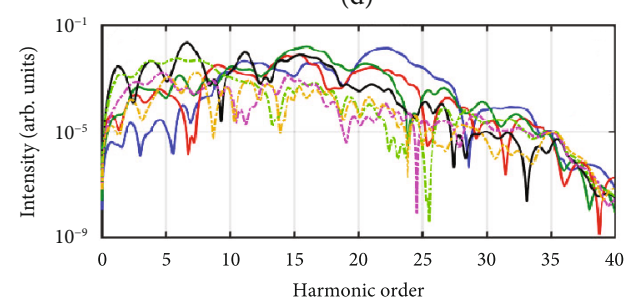

$-\Delta \mathbf{j}=-1$
$-\Delta \mathbf{j}=-2$
$-\Delta \mathbf{j}=-3$

(e)

FIGURE 4: Delocalization of HHG in ZnO. (a) $1 \mathrm{D}$ conduction and valence band structures along the $\Gamma$-M direction in $\mathrm{ZnO}$ adopted in Reference [23]. The time-dependent position (b) and velocity (c) of electron (black) and hole (red) trajectories with initial ionization time at $-0.046 T_{0}$. (d) The distance between the ionization and recombination positions of the electron-hole pair as a function of ionization (dashed line) and recombination (solid line) instants, respectively. (e) The Wannier analysis with the same laser and material parameters as in (d).

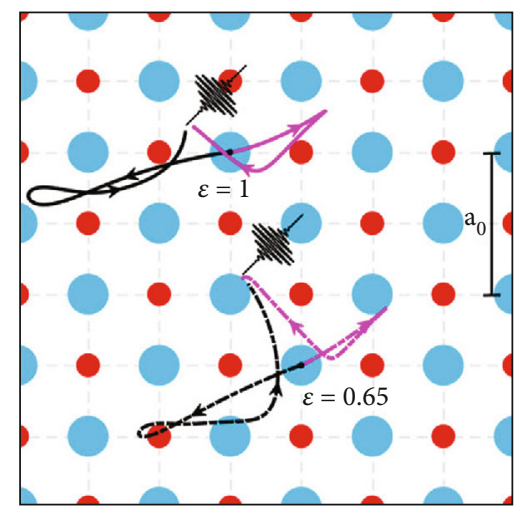

FIGURE 5: Electron (black) and hole (magenta) trajectories for circular $|\varepsilon|=1$ (solid lines) and elliptical polarization $|\varepsilon|=0.65$ (dot-dashed lines). The major axes of the polarization direction are set along the $\mathrm{Mg}-\mathrm{O}$ bonding direction and the $\mathrm{O}-\mathrm{O}$ (or $\mathrm{Mg}$ $\mathrm{Mg}$ ) direction, respectively.

occur (see Figure 3(d) and Movie S5), which is consistent with both experiments in Reference [21] and our quantum simulations, where the harmonic signal at the high-energy part is weakest along $\theta=27^{\circ}$ (see Figures 1 and 2). Therefore, forward scatterings by side collisions with neighboring atoms, which suppress the electron recombination with its associated hole, is the main underlying physics for the minimum of the HHG signal along this direction. It should be mentioned that for a laser polarization along $\theta=0^{\circ}, 90^{\circ}, 18$ $0^{\circ}$, and $270^{\circ}$, the electron/hole itself moves along the line $k_{x / y}=0$, where $\nabla_{k_{x}} E_{c / v}(k)=0$ or $\nabla_{k_{y}} E_{c / v}(k)=0$, so it is uncorrelated to the collision/scattering by other neighboring atoms even though it might cross same kind of critical lines (see the solid lines which cross the $\Gamma$ point in Figures 3(a) and $3(\mathrm{~b})$ ).

When driven by a circularly or elliptically polarized laser, such forward scatterings by side collisions with other neighboring atoms could indeed help the electron reencounter with its associated hole (see Figure 5) (more details can be seen in Movie S6 and Movie S7 in Supplementary Materials). This is again consistent with the measured ellipticity dependence of $\mathrm{HHG}$ in $\mathrm{MgO}$ [21], where harmonics at high-energy part have maxima at circular polarization $|\varepsilon|=1$ where the major axis is set along the Mg-O bonding direction and at elliptical polarization around $|\varepsilon|$ $=0.65$ where major axis along $\mathrm{O}-\mathrm{O}$ (or $\mathrm{Mg}-\mathrm{Mg}$ ) direction.

\section{Conclusions}

We propose and establish a transparent collision and recombination picture for interband HHG in semiconductors by providing a direct mapping between the band structure and the collision dynamics. If after the ionization the electron and hole move only within a small fraction of the Brillouin zone near the $\Gamma$ point, practically, no scattering of electrons and holes with other atomic sites is possible. In this case, the electron and its associated hole are driven back together by the laser field, a mechanism quite similar to the atomic case. Therefore, the HHG spectrum shows a weak anisotropy.

For high field amplitudes and ionization occurring before the field crest, the electron can reach larger $k$-points in momentum space and, correspondingly, it can collide in real space with other atomic sites in the periodic array. This collision information can be directly read out from the band structure. Head-on collisions, which induce a direction 
reversal, occur at the Van Hove singularities where $\nabla_{k}\left(E_{c, v}(k)\right)=0$. Such collisions may lead to the recombination of electron-hole pairs without the need of a sign reversal of the electric field, which is a novel HHG mechanism that is absent in the atomic case. When the electron/hole passes through the critical lines where only $\nabla_{k_{x}} \varepsilon(k)=0$ or $\nabla_{k_{y}} \varepsilon(k)$ $=0$, side collision occurs in the real-space trajectories. Such collisions support electron-hole recombination for the case of circular/elliptical polarization; however, these diminish the electron-hole recombination for linear polarization.

In summary, our electron-hole recombination picture gives an unified and entirely new explanation of many aspects of previous experimental observations and theoretical findings $[14,21,23,30]$ and paves the way towards a more complete understanding of the underlying collision dynamics within subcycle attosecond time scale in solids.

\section{Data Availability}

The data that support the findings of this study are available from the corresponding authors upon reasonable request.

\section{Conflicts of Interest}

The authors declare that there is no conflict of interest regarding the publication of this article.

\section{Authors' Contributions}

X. Song, T. Meier, and W. Yang developed the idea. R. Zuo, A. Trautmann, G. Wang, W. Hannes, and S. Yang performed the simulations and analyzed the data. All authors contributed to the interpretation of the results and the preparation of the manuscript and reviewed the manuscript.

\section{Acknowledgments}

We thank S. Jiang and R. Lu for their helpful discussions. We thank the PC ${ }^{2}$ (Paderborn Center for Parallel Computing) for providing computing time. This work was supported by the National Natural Science Foundation of China (Grant No. 12074240, No. 91950101, and No. 11774215), the Deutsche Forschungsgemeinschaft (DFG, German Research Foundation) (project number 231447078 TRR 142) (project A07), the Sino-German Mobility Programme (Grant No. M-0031), the Department of Education of Guangdong Province (Grant No. 2018KCXTD011), and the Open Fund of the State Key Laboratory of High Field Laser Physics (SIOM).

\section{Supplementary Materials}

Houston state-based semiconductor Bloch equations (SBEs), classical trajectory model for solid-state systems, Wannier state-based picture, transition dipole moment of $\mathrm{MgO}$, and movies of trajectories of electron and hole in both real and moment spaces. Figure S1: the imaginary part of dipole matrix element shown in the first $\mathrm{BZ}$ for transition from $\mathrm{VB}$ to $\mathrm{CB}$ used in the main text. Movie S1: trajectories of electron and hole starting at $t=-0.13 T_{0}$ for the laser polarization along $\Theta=0^{\circ}$ in MgO. Movie S2: trajectories of electron and hole starting at $t=-0.18 T_{0}$ for the laser polarization along $\Theta=$ $45^{\circ}$ in MgO. Movie S3: trajectories of electron and hole starting at $t=0.13 T_{0}$ for the laser polarization along $\Theta=0^{\circ}$ in $\mathrm{MgO}$. Movie S4: trajectories of electron and hole starting at $t=-0.046 T_{0}$ for the laser polarization along $\Gamma-\mathrm{M}$ in $\mathrm{ZnO}$. Movie S5: trajectories of electron and hole starting at $t=-0.13 T_{0}$ for the laser polarization along $\Theta=27^{\circ}$ in $\mathrm{MgO}$. Movie S6: trajectories of electron and hole starting at $t=0.015 T_{0}$ when driven by elliptically polarized laser field in MgO. Movie S7: trajectories of electron and hole starting at $t=-0.105 T_{0}$ when driven by circularly polarized laser field in $\mathrm{MgO}$. (Supplementary Materials)

\section{References}

[1] J. Li, J. Lu, A. Chew et al., "Attosecond science based on high harmonic generation from gases and solids," Nature Communications, vol. 11, no. 1, p. 2748, 2020.

[2] F. Krausz and M. Ivanov, "Attosecond physics," Reviews of Modern Physics, vol. 81, pp. 163-234, 2009.

[3] P. B. Corkum and F. Krausz, "Attosecond science," Nature Physics, vol. 3, pp. 381-387, 2007.

[4] P. B. Corkum, "Plasma perspective on strong field multiphoton ionization," Physical Review Letters, vol. 71, no. 13, pp. 1994-1997, 1993.

[5] P. B. Corkum, "Recollision physics," Physics Today, vol. 64, no. 3, pp. 36-41, 2011.

[6] J. Itatani, J. Levesque, D. Zeidler et al., “Tomographic imaging of molecular orbitals," Nature, vol. 432, no. 7019, pp. 867-871, 2004.

[7] R. Torres, N. Kajumba, J. G. Underwood et al., "Probing orbital structure of polyatomic molecules by high-order harmonic generation," Physical Review Letters, vol. 98, no. 20, article 203007, 2007.

[8] W. Li, X. Zhou, R. Lock et al., "Time-resolved dynamics in $\mathrm{N}_{2} \mathrm{O}_{4}$ probed using high harmonic generation," Science, vol. 322, no. 5905, pp. 1207-1211, 2008.

[9] C. Vozzi, M. N. F. Calegari, G. Sansone, M. Nisoli, S. D. Silvestri, and S. Stagira, "Generalized molecular orbital tomography," Nature Physics, vol. 7, no. 10, pp. 822-826, 2011.

[10] D. Shar, H. Soifer, B. D. Bruner et al., "Resolving the time when an electron exits a tunnelling barrier," Nature, vol. 485, no. 7398, pp. 343-346, 2012.

[11] H. G. Kurz, M. Kretschmar, T. Binhammer et al., "Revealing the microscopic real-space excursion of a laser-driven electron," Physical Review X, vol. 6, no. 3, article 031029, 2016.

[12] S. Ghimire, A. D. DiChiara, E. Sistrunk, P. Agostini, L. F. DiMauro, and D. A. Reis, "Observation of high-order harmonic generation in a bulk crystal," Nature Physics, vol. 7, no. 2, pp. 138-141, 2011.

[13] B. Zaks, R. B. Liu, and S. Sherwin, "Experimental observation of electron-hole recollisions," Nature, vol. 483, no. 7391, pp. 580-583, 2012.

[14] G. Vampa, T. J. Hammond, N. Thiré et al., "Linking high harmonics from gases and solids," Nature, vol. 522, p. $462,2015$.

[15] G. Vampa, C. R. McDonals, G. Orlando, D. D. Klug, P. B. Korkum, and T. Brabec, "Theoretical analysis of highharmonic generation in solids," Physical Review Letters, vol. 113, no. 7, article 073901, 2014. 
[16] G. Vampa, C. R. McDonals, G. Orlando, P. B. Corkum, and T. Brabec, "Semiclassical analysis of high harmonic generation in bulk crystals," Physical Review B, vol. 91, article 064302, 2015.

[17] F. Langer, M. Hohenleutner, C. P. Schmid et al., "Lightwavedriven quasiparticle collisions on a subcycle timescale," Nature, vol. 533, p. 225, 2016.

[18] G. Ndabashimiye, S. Ghimire, M. Wu et al., "Solid-state harmonics beyond the atomic limit," Nature, vol. 534, no. 7608, pp. 520-523, 2016.

[19] X. Song, S. Yang, R. Zuo, T. Meier, and W. Yang, "Enhanced high-order harmonic generation in semiconductors by excitation with multicolor pulses," Physical Review A, vol. 101, article 033410, 2020

[20] X. Song, R. Zuo, S. Yang, P. Li, T. Meier, and W. Yang, "Attosecond temporal confinement of interband excitation by intraband motion," Optics Express, vol. 27, no. 3, pp. 2225-2234, 2019.

[21] Y. S. You, D. A. Reis, and S. Ghimire, "Anisotropic highharmonic generation in bulk crystals," Nature Physics, vol. 13, no. 4, pp. 345-349, 2017.

[22] Y. S. You, E. Cunningham, D. A. Reis, and S. Ghimire, "Probing periodic potential of crystals via strong-field re-scattering," Journal Physics B-Atomic Molecular and Optical Physics, vol. 51, article 114002, 2018.

[23] E. N. Osika, A. Chacón, L. Ortmann et al., "Wannier-Bloch approach to localization in high-harmonics generation in solids," Physical Review X, vol. 7, no. 2, article 021017, 2017.

[24] L. Liu, J. Zhao, W. Dong, J. Liu, L. Huang, and Z. Zhao, "Spatial coherence in high-order harmonic generation from periodic solid structures," Physical Review A, vol. 96, no. 5, article 053403, 2017.

[25] L. Li, P. Lan, X. Zhu et al., "Reciprocal-space-trajectory perspective on high-harmonic generation in solids," Physical Review Letters, vol. 122, no. 19, article 193901, 2019.

[26] X. Zhang, J. Li, Z. Zhou et al., "Ellipticity dependence transition induced by dynamical Bloch oscillations," Physical Review $B$, vol. 99, no. 1, article 014304, 2019.

[27] L. Yue and M. B. Gaarde, "Imperfect recollisions in highharmonic generation in solids," Physical Review Letters, vol. 124 , no. 15 , p. 153204, 2020.

[28] S. Y. Kruchinin, F. Krausz, and V. S. Yakovlev, "Strong-field phenomena in periodic systems," Review of Modern Physics, vol. 90, no. 2, article 021002, 2018.

[29] U. Huttner, M. Kira, and S. W. Koch, "Ultrahigh off-resonant field effects in semiconductors," Laser Photonics Reviews, vol. 11, no. 4, p. 1700049, 2017

[30] A. J. Uzan, G. Orenstein, A. Jimenez-Galan et al., "Attosecond spectral singularities in solid-state high-harmonic generation," Nature Photonics, vol. 14, p. 183, 2020.

[31] D. Golde, T. Meier, and S. W. Koch, "High harmonics generated in semiconductor nanostructures by the coupled dynamics of optical inter- and intraband excitations," Physical Review $B$, vol. 77, no. 7, article 075330, 2008.

[32] http://elk.sourceforge.net/.

[33] L. Yue and M. B. Gaarde, "Structure gauges and laser gauges for the semiconductor Bloch equations in high-order harmonic generation in solids," Physical Review A, vol. 101, no. 5 , article $053411,2020$.
[34] D. Vanderbilt, Berry phase in electronic structure theory: electric polarization, orbital magnetization and topological insulators, Cambridge University Press, Cambridge, England, 2018.

[35] S. Jiang, C. Yu, J. Chen, Y. Huang, R. Lu, and C. D. Lin, "Smooth periodic gauge satisfying crystal symmetry and periodicity to study high-harmonic generation in solids," Physical Review B, vol. 102, no. 15, p. 155201, 2020.

[36] X. Chai, X. Ropagnol, S. M. Raeis-Zadeh, M. Reid, S. SafaviNaeini, and T. Ozaki, "Subcycle terahertz nonlinear optics," Physical Review Letters, vol. 121, no. 14, p. 143901, 2018.

[37] M. T. Hassan, T. T. Luu, A. Moulet et al., "Optical attosecond pulses and tracking the nonlinear response of bound electrons," Nature, vol. 530, no. 7588, pp. 66-70, 2016.

[38] H. Liang, P. Krogen, Z. Wang et al., "High-energy midinfrared sub-cycle pulse synthesis from a parametric amplifier," Nature Communications, vol. 8, no. 1, p. 141, 2017.

[39] M. Wu, Y. You, S. Ghimire et al., "Orientation dependence of temporal and spectral properties of high-order harmonics in solids," Physical Review A, vol. 96, article 063412, 2017.

[40] N. Yoshikawa, K. Nagai, K. U. Y. Takaguchi, S. Sasaki, Y. Miyata, and K. Tanaka, "Interband resonant highharmonic generation by valley polarized electron-hole pairs," Nature Communications, vol. 10, no. 1, p. 3709, 2019.

[41] L. V. Hove, "The occurrence of singularities in the elastic frequency distribution of a crystal," Physical Review, vol. 89, no. 6, pp. 1189-1193, 1953.

[42] D. Brust, J. C. Phillips, and F. Bassani, "Critical points and ultraviolet reflectivity of semiconductors," Physical Review Letters, vol. 9, no. 3, pp. 94-97, 1962.

[43] A. Beiser, Concepts of Modern Physics, McGraw-Hill, New York, 6th edition, 2003. 